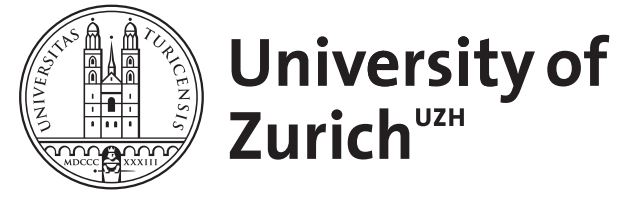

Zurich Open Repository and Archive

University of Zurich

University Library

Strickhofstrasse 39

CH-8057 Zurich

www.zora.uzh.ch

Year: 2014

\title{
Renale Kontrolle der Kaliumhomöostase
}

Loffing, J

DOI: https://doi.org/10.1007/s11560-014-0863-3

Posted at the Zurich Open Repository and Archive, University of Zurich

ZORA URL: https://doi.org/10.5167/uzh-171994

Journal Article

Published Version

Originally published at:

Loffing, J (2014). Renale Kontrolle der Kaliumhomöostase. Der Nephrologe, 9(6):473-476.

DOI: https://doi.org/10.1007/s11560-014-0863-3 
Nephrologe 2014 · 9:473-476

DOI 10.1007/s11560-014-0863-3

Online publiziert: 12. November 2014

c) Springer-Verlag Berlin Heidelberg 2014

\section{Redaktion}

A. Kurtz, Regensburg

\section{J. Loffing}

Anatomisches Institut, Universität Zürich, Schweiz

\section{Physiologische Bedeutung von Kalium}

Kalium $\left(\mathrm{K}^{+}\right)$spielt eine fundamentale Rolle bei einer Vielzahl biologischer Prozesse wie der Regulierung des Zellvolumens, des intrazellulären $\mathrm{pH}$-Werts, der DNAund Proteinbiosynthese sowie des zellulären Membranpotenzials. Während die intrazelluläre Konzentration von $\mathrm{K}^{+}$hoch ist (ca. $120 \mathrm{mM}$ ), ist die extrazelluläre Konzentration gering (ca. $4 \mathrm{mM}$ ). Dieses Konzentrationsgefälle ist entscheidend für das Ruhemembranpotenzial und damit für eine normale Funktion von erregbaren Zellen in Nervengewebe, Skelett- und Herzmuskel. Entgleisungen der $\mathrm{K}^{+}$-Homöostase führen entsprechend $\mathrm{zu}$ schwerwiegenden Funktionsstörungen einschließlich lebensbedrohlicher Herzrhythmusstörungen.

Der größte Teil (ca. 98\%) des Gesamtkörperkaliums (ca. $3500 \mathrm{mmol}$ ) ist im Intrazellularraum gespeichert. Nur ein sehr kleiner Teil (70-100 mmol) befindet sich im Extrazellularraum. Eine erwachsene Person nimmt pro Tag durchschnittlich die gleiche Menge $\mathrm{K}^{+}$auf, wie im Extrazellularraum vorhanden ist. Dass es im Anschluss an eine $\mathrm{K}^{+}$-reiche Mahlzeit nicht zu einem gefährlichen Anstieg der extrazellulären $\mathrm{K}^{+}$-Konzentration kommt, beruht im Wesentlichen auf einer raschen Verschiebung von $\mathrm{K}^{+}$aus dem Extra- in den Intrazellularraum und einem Anstieg der $\mathrm{K}^{+}$-Ausscheidung im Urin. Insgesamt werden rund $90 \%$ des aufgenommen $\mathrm{K}^{+}$ über die Nieren und 10\% über den Darm ausgeschieden. Die Mechanismen der renalen $\mathrm{K}^{+}$-Ausscheidung werden im Weiteren kurz beschrieben (weitere Details in [1]).

\section{Zelluläre und molekulare Mechanismen der Kaliumresorption und -sekretion entlang des Nephrons}

Die Nieren kontrollieren die $\mathrm{K}^{+}$-Homöostase durch eine Kombination von $\mathrm{K}^{+}$-Filtration, -Resorption und -Sekretion entlang des Nephrons (• Abb. 1). Das im Blut gelöste $\mathrm{K}^{+}$wird zunächst glomerulär frei filtriert und dann zu etwa $80 \%$ entlang des proximalen Tubulus (PT) parazellulär resorbiert. Das $\mathrm{K}^{+}$folgt dabei passiv der Resorption von Salz und Wasser. Zum Ende des proximalen Tubulus unterstützt eine zunehmende Elektropositivität des Tubuluslumens den Rücktransport der $\mathrm{K}^{+}$-Ionen. Während im dünnen absteigenden Teil der Henle-Schleife $\mathrm{K}^{+}$sezerniert wird, wird es im aufsteigenden Teil der Henle-Schleife wieder resorbiert. Dieses $\mathrm{K}^{+}$-Recycling im Nierenmark trägt wesentlich zur hohen interstitiellen $\mathrm{K}^{+}$-Konzentration in der Nierenpapille bei. Die Resorption von $\mathrm{K}^{+}$ im dicken aufsteigenden Teil der Schleife (,thick ascending limb“, TAL) erfolgt dabei sowohl aktiv transzellulär über den furosemidsensitiven Na-K-2Cl-Kotransporter als auch passiv parazellulär durch die Elektropositivität des Tubuluslumens. Entlang der Henle-Schleife werden so etwa weitere $10 \%$ des filtrierten $\mathrm{K}^{+}$resorbiert, sodass insgesamt nur noch rund $10 \%$ des filtrierten $\mathrm{K}^{+}$die Macula densa und die ihr nachgeschalteten Tubulusabschnitte erreichen [Pars convoluta des distalen Tubulus, "distal convoluted tubule“ (DCT); Verbindungstubulus, „connecting tubule" (CNT); Sammelrohr, „collecting duct" (CD), mit kortikalem (CCD) und medullären (MCD) Abschnitten]. Während in PT und Henle-Schleife $\mathrm{K}^{+}$weit- gehend unreguliert resorbiert wird, stellt der Organismus die $\mathrm{K}^{+}$-Transport-Rate in DCT, CNT und CCD sehr genau ein. Unter einer $\mathrm{K}^{+}$-armen Diät wird $\mathrm{K}^{+}$resorbiert, während unter einer $\mathrm{K}^{+}$-haltigen Diät $\mathrm{K}^{+}$sezerniert wird. Bei einer $\mathrm{K}^{+}$reichen Ernährung kann hier sogar mehr $\mathrm{K}^{+}$sezerniert werden, als glomerulär filtriert wurde.

Die Mechanismen der $\mathrm{K}^{+}$-Resorption und -Sekretion entlang des DCT, CNT und $\mathrm{CD}$ sind komplex und an die Funktion verschiedener Tubulusepithelzellen gekoppelt. Neben den segmentspezifischen DCT-, CNT- und CD-Zellen beteiligen sich daran auch die sog. Schaltzellen, die vom Endabschnitt des DCT (DCT2) bis zum Anfangsteil des medul-

\begin{tabular}{|c|c|}
\hline $\begin{array}{l}\mathrm{K}^{+} \text {-Aussscheidung } \\
\text { wird erhöht }\end{array}$ & $\begin{array}{l}\mathrm{K}^{+}-\text {Ausscheidung } \\
\text { wird gesenkt }\end{array}$ \\
\hline $\mathrm{K}^{+}$-reiche Ernährung & $\mathrm{K}^{+}$-arme Ernährung \\
\hline \multicolumn{2}{|l|}{ Aldosteron } \\
\hline Glukokortikoide & Progesteron \\
\hline Vasopressin & Noradrenalin \\
\hline \multicolumn{2}{|l|}{ Insulin } \\
\hline \multicolumn{2}{|l|}{ Harnflussrate } \\
\hline Alkalose & Azidose \\
\hline Schleifendiuretika & $\begin{array}{l}\mathrm{K}^{+} \text {-sparende Diuretika } \\
\text { (z. B. Amilorid) }\end{array}$ \\
\hline \multirow[t]{3}{*}{ Thiazide } & $\begin{array}{l}\text { MR-Antagonisten } \\
\text { (z. B. Spironolacton) }\end{array}$ \\
\hline & $\begin{array}{l}\text { AT1-Antagonisten } \\
\text { (z. B. Losartan) }\end{array}$ \\
\hline & Trimethoprim \\
\hline \multicolumn{2}{|l|}{ Bartter-Syndrom } \\
\hline \multicolumn{2}{|l|}{ Gitelman-Syndrom } \\
\hline Liddle-Syndrom & $\begin{array}{l}\text { Pseudohypoaldo- } \\
\text { steronismus Typ } 1\end{array}$ \\
\hline $\begin{array}{l}\text { MR Mineralokortikoidr } \\
\text { Rezeptor-Subtyp } 1 .\end{array}$ & ptor, AT1 Angiotensin-II- \\
\hline
\end{tabular}



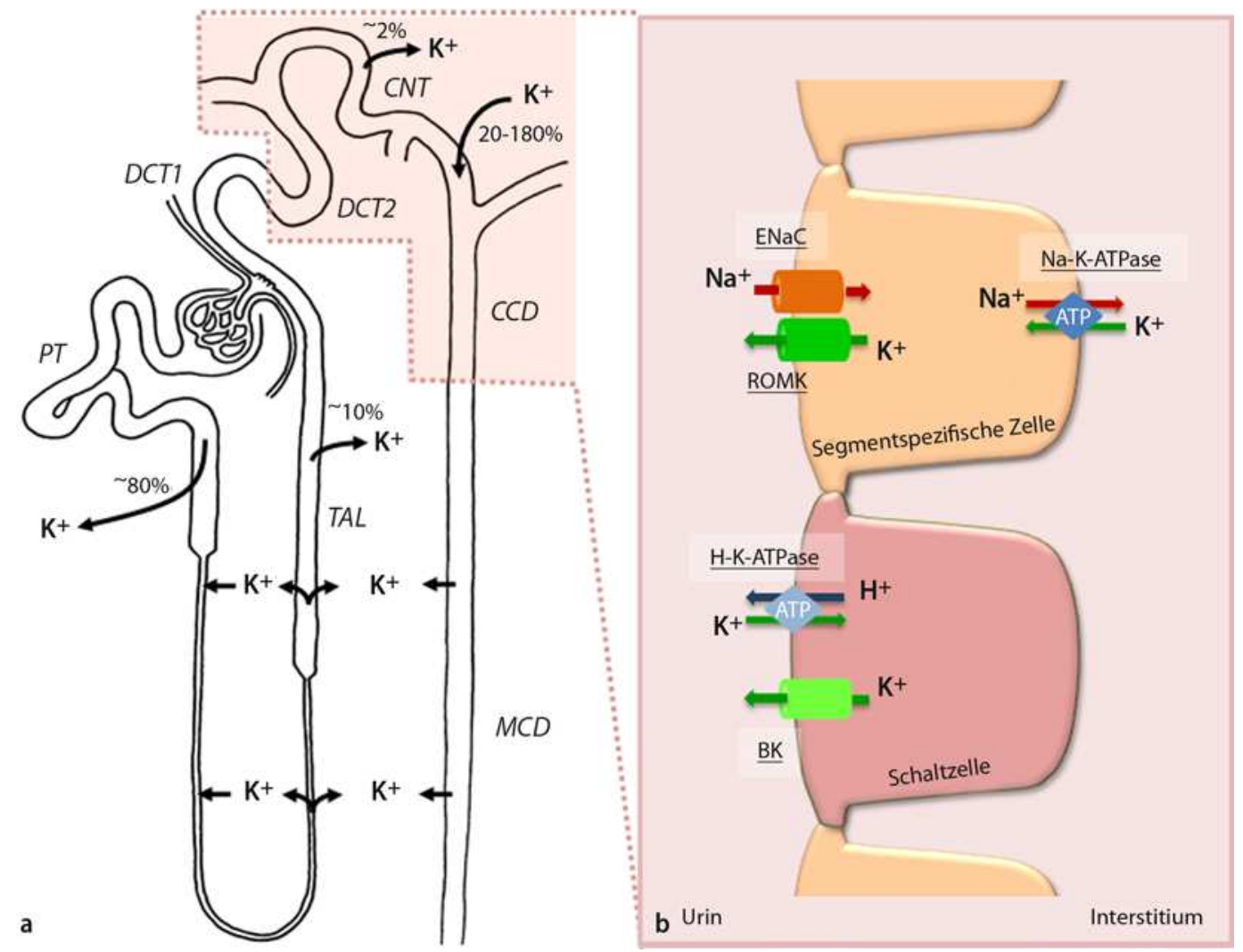

Abb. $1<$ a Schematische Darstellung des $\mathrm{K}^{+}$-Transports entlang des $\mathrm{Ne}-$ phrons; rot hervorgehoben sind die Tubulusabschnitte, in denen in Abhängigkeit vom $\mathrm{K}^{+}$-Gehalt der Nahrung $\mathrm{K}^{+}$resorbiert oder ausgeschieden wird. b Schematische Darstellung der segmentspezifischen Zellen und Schaltzellen in DCT2, CNT, CCD mit Ionenkanälen und ATPasen, die für $\mathrm{K}^{+}$-Sekretion bzw. -Resorption wichtig sind

lären Sammelrohrs zwischen die eigentlichen DCT-, CNT-und CD-Zellen eingestreut sind. Die DCT-Zellen haben in der Regel noch eine relativ geringe $\mathrm{K}^{+}$-Transport-Rate. In DCT2, CNT und im Anfangsteil des CCD ist die Befähigung zur Steigerung der $\mathrm{K}^{+}$-Sekretion am größten. Die $\mathrm{K}^{+}$-Sekretion erfolgt hier zum einen über den ROMK-K ${ }^{+}$-Kanal, der in der luminalen Membran der DCT2-, CNT- und CCD-Zellen vorkommt, und zum anderen über den $\mathrm{BK}-\mathrm{K}^{+}$-Kanal, der in der luminalen Membran von Schaltzellen nachgewiesen wurde. Die $\mathrm{K}^{+}$-Sekretion wird durch ein lumennegatives elektrochemisches Potenzial getrieben. Dieses wird durch die $\mathrm{Na}^{+}$-Resorption über den amiloridsensitiven epithelialen Natriumkanal $(\mathrm{ENaC})$ in DCT2, CNT und CD verursacht. Die Fähigkeit zur $\mathrm{K}^{+}$-Sekretion nimmt entlang des CD ab. Als Folge der $\mathrm{K}^{+}$-Sekretion und der vasopressingetriebenen Wasserresorption steigt die intratubuläre $\mathrm{K}^{+}$-Konzentration entlang des $\mathrm{CD}$ an, sodass im MCD der transepitheliale Gradient wieder in Richtung $\mathrm{K}^{+}$-Resorption verschoben ist. Zusätzlich kann eine apikale, $\mathrm{K}^{+}$-resorbierende $\mathrm{H}$-K-ATPase in
Schalt- und MCD-Zellen die renale $\mathrm{K}^{+}$Ausscheidung modulieren.

\section{Regulierung der renalen Kaliumausscheidung}

Die renale $\mathrm{K}^{+}$-Ausscheidung wird durch eine Vielzahl von Faktoren reguliert (- Tab. 1). Einer der wichtigsten Faktoren ist der $\mathrm{K}^{+}$-Gehalt der Nahrung $[1,2]$. Die gesunden Nieren passen die renale $\mathrm{K}^{+}$-Ausscheidung genau an die $\mathrm{K}^{+}$-Aufnahme an. Eine bedeutende Rolle wird hierbei Aldosteron zugeschrieben, welches genau in den Tubuli wirkt (DCT2, CNT und CD), in denen $\mathrm{K}^{+}$aktiv sezerniert wird. Aldosteron steigert hier über eine Aktivierung von $\mathrm{ENaC}$ die elektrochemische Triebkraft für die $\mathrm{K}^{+}$-Sekretion. Allerdings tritt die Kaliurese nach einer $\mathrm{K}^{+}$-reichen Diät vielfach bereits vor einem Anstieg der Aldosteronspiegel auf. Außerdem zeigen auch adrenalektomierte Tiere noch die Fähigkeit, die renale $\mathrm{K}^{+}$-Auscheidung an eine $\mathrm{K}^{+}$-Beladung anzupassen. Neben Aldosteron stimulieren zahlreiche weitere Hormone wie Glukokortikoide, Vasopressin und Insulin $\mathrm{ENaC}$ und die renale $\mathrm{K}^{+}$-Aus- scheidung. Umgekehrt senken Progesteron und Noradrenalin die renale $\mathrm{K}^{+}$Ausscheidung. Progesteron stimuliert die H-K-ATPase. Noradrenalin erhöht die Aufnahme von $\mathrm{K}^{+}$in extrarenale Zellen und senkt so die glomeruläre Filtration von $\mathrm{K}^{+}$. Zusätzlich wird die renale $\mathrm{K}^{+}$-Sekretion gehemmt. Eine erhöhte Harnflussrate aktiviert BK-Kanäle und erhöht durch das Ausschwemmen von $\mathrm{K}^{+}$ den chemischen Gradienten für die $\mathrm{K}^{+}$Sekretion. Alkalose und Azidose haben gegensätzliche Effekte, die u. a. durch direkte Wirkungen des veränderten intrazellulären $\mathrm{pH}$-Werts auf ROMK vermittelt werden. Diuretika und genetische Erkrankungen, die den Salztransport in TAL (Schleifendiuretika bzw. BartterSyndrom) oder DCT (Thiazide bzw. Gitelman-Syndrom) blockieren, erhöhen die renale $\mathrm{K}^{+}$-Ausscheidung durch die erhöhte Harnflussrate und eine kompensatorische, aldosteronvermittelte Aktivierung von $\mathrm{ENaC}$. Umgekehrt führt eine Funktionseinschränkung von $\mathrm{ENaC}$ durch Diuretika (Amilorid), Antibiotika (Trimethoprim), Mineralokortikoid- oder Angiotensinrezeptorblocker (Spironolacton, Losartan) sowie genetische Erkrankun- 


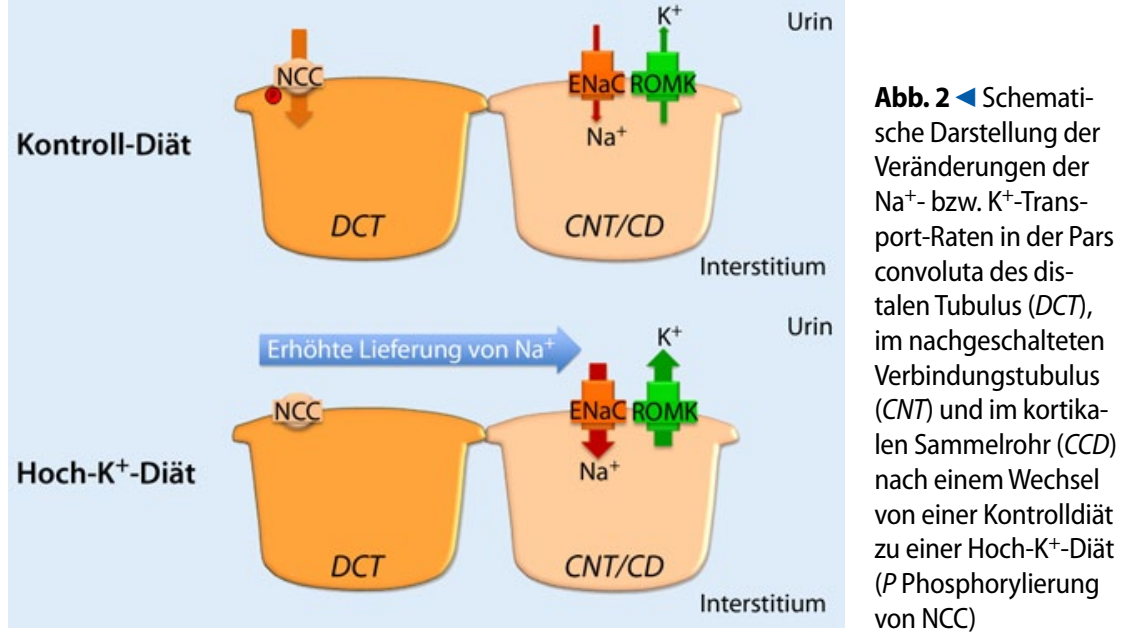

gen (Pseudohypoaldosteronismus Typ 1) zu einer reduzierten renalen $\mathrm{K}^{+}$-Ausscheidung.

Die molekularen Mechanismen, die ROMK, BK, ENaC und H-K-ATPase in der luminalen Membran des distalen Tubulus und des Sammelrohrs regulieren, sind noch weitgehend unbekannt. Eine wichtige Rolle scheinen die aldosteronabhängige Kinase Sgk1 und andere Kinasen wie MAPK, WNK1, WNK4, PKA und PKC sowie Ubiquitinligasen zu spielen [2].

\section{Aldosteronparadoxon}

Eine $\mathrm{K}^{+}$-reiche Ernährung erhöht die Plasmaaldosteronspiegel und die ENaCvermittelte $\mathrm{Na}^{+}$-Resorption in DCT2, $\mathrm{CNT}$ und CD. Interessanterweise kommt es im Anschluss an eine $\mathrm{K}^{+}$-reiche Mahlzeit aber nicht zu einer renalen $\mathrm{Na}^{+}-\mathrm{Re}-$ tention, sondern $\mathrm{zu}$ einer Natriurese. Auch erhöht eine $\mathrm{K}^{+}$-reiche Ernährung in der Regel nicht den Blutdruck, sondern wirkt antihypertensiv. Die Gründe für dieses Aldosteronparadoxons sind unklar. In Mäusen inaktiviert eine $\mathrm{K}^{+}$-reiche Diät den thiazidsensitiven $\mathrm{NaCl}-\mathrm{Ko}$ transporter (NCC) im DCT über eine rasche Dephosphorylierung [3]. Dies erhöht die Harnflussrate und die $\mathrm{Na}^{+}-\mathrm{Be}$ ladung in den nachgeschalteten Segmenten (CNT und CD). Durch die vermehrte Bereitstellung von $\mathrm{Na}^{+}$im Tubuluslumen werden die $\mathrm{ENaC}$-vermittelte $\mathrm{Na}^{+}$Resorption und damit die elektrochemische Triebkraft für die $\mathrm{K}^{+}$-Sekretion gesteigert (• Abb. 2). Dieser thiazidähnli- che Effekt verbessert vermutlich nicht nur die renale $\mathrm{K}^{+}$-Ausscheidung, sondern erklärt zumindest teilweise auch die natriuretischen und antihypertensiven Effekte einer $\mathrm{K}^{+}$-reichen Ernährung.

\section{Ausblick}

Während die tubulären Transportmechanismen für $\mathrm{K}^{+}$weitgehend bekannt sind, sind wesentliche Fragen zur renalen Kontrolle der $\mathrm{K}^{+}$-Homöostase weiterhin offen. So ist unklar, wo und wie im Körper eine veränderte $\mathrm{K}^{+}$-Konzentration in der Nahrung oder im Blutplasma erfasst wird. Auch die Wege, über die diese Informationen an die Tubulusepithelzellen vermittelt werden, und welche Signaltransduktionskaskaden die beteiligten Ionentransporter und -kanäle in der Niere regulieren, sind noch wenig erforscht. Kenntnis der zugrunde liegenden Mechanismen könnte dazu beitragen, Störungen der $\mathrm{K}^{+}$Homöostase zu vermeiden und besser $\mathrm{zu}$ behandeln und die antihypertensiven Effekte einer $\mathrm{K}^{+}$-reichen Ernährung gezielt zu nutzen.

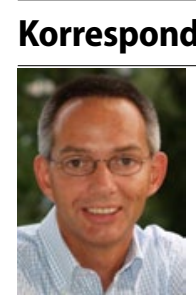

Prof. Dr. J. Loffing

Anatomisches Institut, Universität Zürich

Winterthurerstr. 190,

CH-8057 Zürich, Schweiz

johannes.loffing@

anatom.uzh.ch

\section{Einhaltung ethischer Richtlinien}

Interessenkonflikt. J. Loffing gibt an, dass kein Interessenkonflikt besteht.

Dieser Beitrag beinhaltet keine Studien an Menschen oderTieren.

\section{Literatur}

1. Giebisch G, Windhager E (2009). Transport of potassium. In: Boron WF, Boulpaep EL (eds) Medical physiology, $2^{\text {nd }}$ edn. Saunders Elsevier, Philadelphia, pp 821-834

2. Welling PA (2013) Regulation of renal potassium secretion: molecular mechanisms. Semin Nephrol 33:215-228

3. Sorensen MV, Grossmann S, Roesinger M et al (2013) Rapid dephosphorylation of the renal sodium chloride cotransporter in response to oral potassium intake in mice. Kidney Int 83:811-824

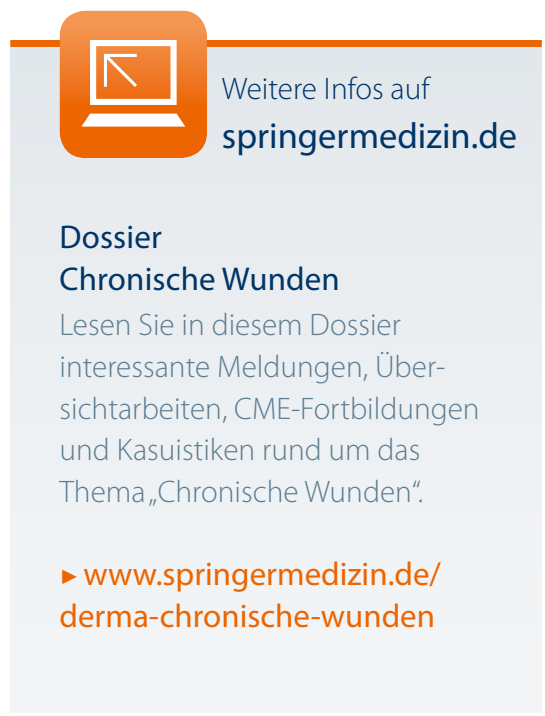




\section{Hier steht eine Anzeige.}

Springer 